\title{
Amygdala activation in maltreated children during pre-attentive emotional processing
}

\author{
Eamon J. McCrory, Stéphane A. De Brito, Philip A. Kelly, Geoffrey Bird, Catherine L. Sebastian,
} Andrea Mechelli, Sophie Samuel and Essi Viding

\section{Background}

Childhood adversity is associated with significantly increased risk of psychiatric disorder. To date, functional magnetic resonance imaging (fMRI) studies of children have mainly focused on institutionalisation and investigated conscious processing of affect.

\section{Aims}

To investigate neural response to pre-attentively presented affect cues in a community sample of children with documented experiences of maltreatment in the home.

\section{Method}

A masked dot-probe paradigm involving pre-attentive presentation of angry, happy and neutral facial expressions was employed. Eighteen maltreated children were compared with 23 carefully matched non-maltreated peers.

\section{Results}

Increased neural response was observed in the right amygdala for pre-attentively presented angry and happy faces in maltreated $v$. non-maltreated children. Level of amygdala activation was negatively associated with age at onset for several abuse subtypes.

\section{Conclusions}

Maltreatment is associated with heightened neural response to positive and negative facial affect, even to stimuli outside awareness. This may represent a latent neural risk factor for future psychiatric disorder.

\section{Declaration of interest}

None.
Childhood maltreatment is a significant risk factor for a wide range of adolescent and adult psychiatric disorders, including anxiety and depression. ${ }^{1,2}$ Although there is good evidence for alterations in psychological functioning following child abuse in the home $e^{1}$ there remains a paucity of neuroimaging studies that have interrogated its impact on brain function. ${ }^{3-6}$ Such studies have the potential to identify prodromal indicators of psychiatric disorder; in other words, pinpoint latent neurobiological risk markers that may prefigure disorder-related patterns of neural activity. A particular advantage of techniques such as functional magnetic resonance imaging (fMRI) is its sensitivity in detecting subtle alterations in neural processing that may not be observable with behavioural indices. ${ }^{7}$ To date only one fMRI study with child participants has investigated the impact of maltreatment in the home on emotional processing. ${ }^{4}$ Maltreated children exhibited significantly greater bilateral activation of the anterior insula and of the right amygdala in response to angry faces, suggesting that exposure to abuse in the home is associated with a pattern of altered brain activity to threat comparable with that seen in several anxiety disorders ${ }^{4,8}$ and in soldiers exposed to combat. ${ }^{9}$ Increased amygdala activation to negative facial cues has also been reported in fMRI studies investigating the impact of institutionalisation, ${ }^{10,11}$ which is a relatively severe form of early adversity, typically associated with a broader and more marked set of developmental impairments. ${ }^{12}$ Related findings have been observed in event-related potential (ERP) studies of physically abused children. ${ }^{5,13,14}$ Childhood adversity, therefore, appears to be associated with increased allocation of attentional resources to consciously processed threat cues.

A separate body of work in non-maltreated individuals with anxiety-related disorders has examined brain response to preattentive emotional processing (i.e. processing of stimuli presented outside of conscious awareness). A distributed network of phylogenetically ancient (largely subcortical) brain structures have been implicated in such 'pre-conscious' processing of affect cues, although several of these areas, including the amygdala, are also engaged during conscious processing. ${ }^{15}$ There is now convincing evidence that such processing is altered in anxiety-related disorders. For example, adolescents with generalised anxiety disorder show greater amygdala activation when processing preattentively presented angry faces. ${ }^{16}$ A similar pattern is also observed in adults with anxiety traits ${ }^{17}$ and in patients with post-traumatic stress disorder (PTSD). ${ }^{18,19}$ The current study aimed to investigate functional brain activation in maltreated children during processing of pre-attentively presented facial affect cues ${ }^{16}$ using a dot-probe paradigm. ${ }^{20}$ We hypothesised that, relative to matched peers, they would show heightened neural response to facial expressions of anger but not happiness. It was expected that this selective response to threat cues would be associated with timing and severity of maltreatment experience. Such a pattern would point to differences in the very early stages of threat perception, prior to higher order strategic or regulatory processing, and constitute a possible marker of increased vulnerability to psychiatric disorder.

\section{Method}

\section{Participants}

Two groups of children were recruited from the London area. Children with documented exposure to physical abuse and/or intimate-partner violence who were in a stable home placement and who did not have any intellectual disabilities (maltreated group, $n=18$ ) were recruited via a community Social Services department. Written informed assent was obtained from each child and written informed consent from a parent. Where there was shared parental responsibility, consent was obtained from the child's biological parent if still contactable and from Social Services.

Comparison children (non-maltreated group, $n=23$ ) comparable on age, self-reported Tanner stage, gender, handedness, cognitive ability, socioeconomic status and ethnicity were recruited from 
Table 1 Background characteristics and questionnaire data for non-maltreated and maltreated group ${ }^{a}$

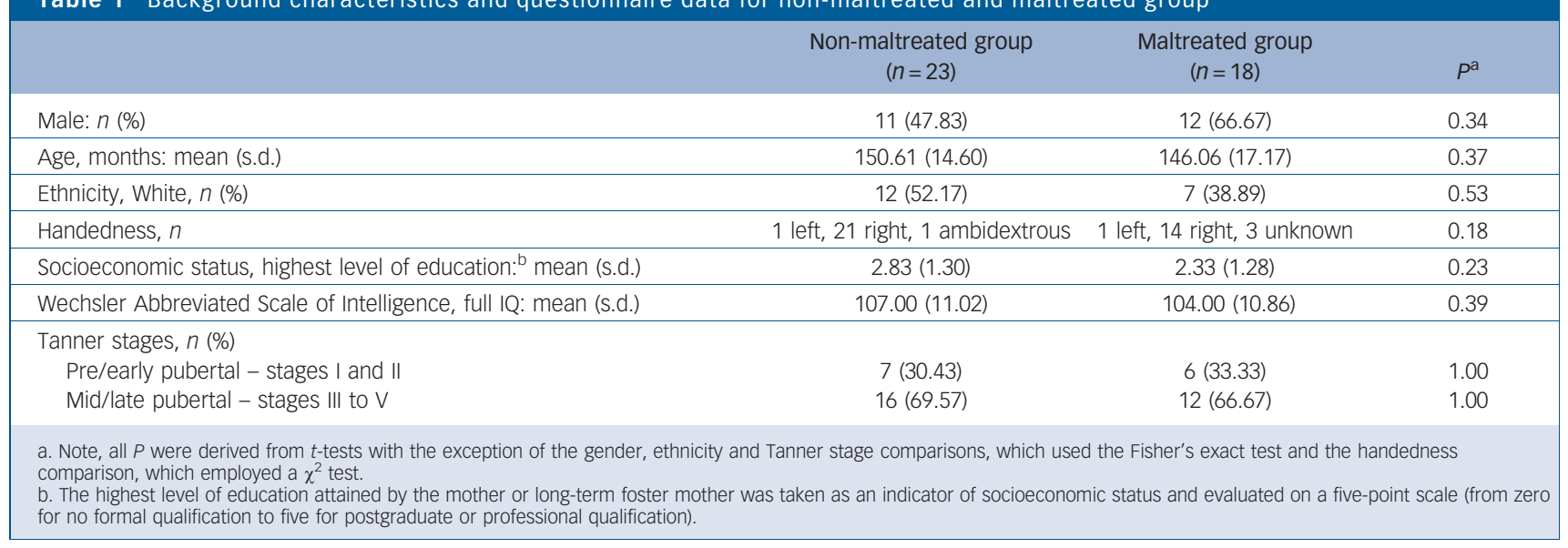

secondary/primary schools and via advertisement in local newspapers and on the internet (Table 1). Exclusion criteria included: history of abuse; history of neglect; exposure to intimate-partner violence as reported by the main carer on the Child Bad Experience Questionnaire ${ }^{21}$ and the Dunedin Abuse Scales; ${ }^{22}$ previous contact with Social Services regarding the child's care. Written informed assent and consent were obtained from the child and a parent(s) respectively. None of the participants reported a history of head trauma, neurological disease, a psychiatric diagnosis, were receiving pharmacological or psychological treatment, or presented with contraindications for MRI. The study was approved by UCL Ethics Committee $(0895 / 002)$

\section{Measures}

\section{Maltreatment history}

Social Services case files were used to obtain an accurate characterisation of a child's maltreatment history. Kaufman and colleagues' coding system ${ }^{23}$ were used for this purpose. This measure allows the severity of four maltreatment subtypes (i.e. physical abuse, sexual abuse, neglect and emotional abuse) to be rated on a scale from zero (not present) to four (severe). Number of cases and mean severity scores for each maltreatment subtype on Kaufman's four-point scale are shown in Table 2, and mean estimated age at onset and duration of each form of abuse was calculated for those participants where this information was available. As expected from a community sample, there was a high degree of overlap among abuse categories. Six cases from the maltreatment group were randomly selected and double-rated by a senior social work professional in relation to each form of abuse; there was $83.3 \%$ agreement in relation to the presence of physical abuse, neglect and sexual abuse and 100\% agreement in relation to emotional abuse.

\section{Child Bad Experience Questionnaire}

Main carers were administered a standardised clinical interview protocol that includes probe questions on bullying, accidents, harsh discipline, physical and sexual abuse. ${ }^{21,24}$

\section{Intimate-partner violence}

To screen for exposure to domestic violence in the non-maltreated group, the Physical Abuse Scale of the Dunedin Abuse Scales ${ }^{22}$ was used to assess specific abusive behaviours from one intimate partner to the other. Respondents answered questions first about their behaviour towards their current or most recent partner and second about the partner's behaviour towards them. The measure yields separate scores for perpetration and victimisation, for both males and females. The Physical Abuse Scale contains all nine items of Straus's Conflict Tactics Scales ${ }^{25}$ (such as slap, choke, beat up), plus four items describing other physically abusive acts (such as twisting arm, bodily throw).

\section{Cognitive ability}

The Vocabulary and Matrix Reasoning subscales of the Wechsler Abbreviated Scales of Intelligence ${ }^{26}$ were used to provide an estimate of full-scale IQ.

\section{Psychiatric symptoms}

The self-report State-Trait Anxiety Inventory for Children $(\text { STAIC })^{27}$ was used to measure state and trait anxiety, and consists of two separate 20-item self-report scales. The Mood and Feelings Questionnaire (MFQ) ${ }^{28}$, a 33-item self-report measure, was used to assess core depressive symptoms. The Trauma Symptom Checklist for Children - A (TSCC-A), ${ }^{29}$ a 44 -item self-report measure, was used to assess acute and chronic post-traumatic symptomatology and other symptom clusters. It includes five clinical scales (anger, anxiety, depression, post-traumatic stress, and dissociation). The Strengths and Difficulties Questionnaire (SDQ) ${ }^{30}$ a 25 -item questionnaire, was completed by parents/

\begin{tabular}{|ll|}
\hline $\begin{array}{l}\text { Table } 2 \text { Abuse subtype severity scores, and estimated } \\
\text { mean age at onset and duration in years }\end{array}$ \\
\hline Abuse subtype & Mean (s.d.) \\
\hline Physical abuse $(n=8)$ & \\
Kaufman score & $1.50(0.54)$ \\
Mean age at onset & $3.29(2.06)$ \\
Mean duration & $5.00(3.16)$ \\
\hline Neglect $(n=16)$ & \\
Kaufman score & $2.63(1.20)$ \\
Mean age at onset & $3.29(3.02)$ \\
Mean duration & $7.00(3.12)$ \\
\hline Sexual abuse $(n=5)$ & \\
Kaufman score & $2.00(1.87)$ \\
Mean age at onset & $5.00(0)$ \\
Mean duration & $2.67(2.08)$ \\
\hline Emotional abuse $(n=17)$ & \\
Kaufman score & $2.88(1.05)$ \\
Mean age at onset & $2.64(3.25)$ \\
Mean duration & $6.27(3.47)$ \\
\hline
\end{tabular}


carers, in order to provide an estimate of emotional symptoms as well as levels of hyperactivity symptoms and conduct problems.

\section{Experimental paradigm}

The fMRI task used identical parameters to those employed by Monk and colleagues. ${ }^{16}$ Briefly, trials started with a $500 \mathrm{~ms}$ fixation cross in the centre of the screen (Fig. 1). Next, two photographs of an actor's face appeared side by side for $17 \mathrm{~ms}$ (pre-attentively). For the face trials, an angry or a happy facial expression was paired with a neutral facial expression of the same actor, while on neutral trials the two photos were identical and showed the actor with a neutral facial expression. Following the brief presentation of the faces, two scrambled faces (the mask) replaced the two faces for $68 \mathrm{~ms}$. The mask was then replaced by an asterisk in one hemifield (on the same side as the emotional face for congruent trials and on the opposite side for incongruent trials) for $1100 \mathrm{~ms}$. Participants indicated which side of the screen the asterisk was displayed on by pressing buttons with the index (indicating left) or middle finger (indicating right) of their dominant hand. Inter-trial intervals were $2300 \mathrm{~ms}$. Previous studies using these parameters show that participants report minimal awareness of details of the briefly presented face stimuli. ${ }^{16,31}$ Eighty actors were each presented twice for a total of 160 trials (i.e. 32 trials for each of the five conditions: angry-neutral congruent, angry-neutral incongruent, happy-neutral congruent, happy-neutral incongruent, neutral-neutral) for each of the two runs. The order of the face trials was randomly determined for each participant. Forty blank trials of the same length as the face trials were also presented randomly. Performance was measured as reaction time and accuracy on the location of the probe. Prior to scanning, participants were trained on the task, but with different stimuli showing neutral faces only.

\section{Image acquisition and analysis}

Participants were scanned using a 1.5 Tesla Siemens (Siemens Medical Systems) Avanto MRI scanner with a 32-channel head coil. A total of 355 multislice $T_{2}{ }^{*}$-weighted echo-planar imaging (EPI) volumes with blood oxygen level-dependent (BOLD) contrast were acquired in two runs of approximately 13 min $30 \mathrm{~s}$ each. The EPI sequence was optimised to reduce BOLD sensitivity losses in the amygdala because of susceptibility artefacts. ${ }^{32}$ Acquisition parameters were: 27 slices per volume; slice thickness $2 \mathrm{~mm}$; gap between slices $1 \mathrm{~mm}$, echo time $(\mathrm{TE})=50 \mathrm{~ms}$; repetition time $(\mathrm{TR})=85.2 \mathrm{~ms}$; slice tilt $-30^{\circ}(\mathrm{T}>\mathrm{C})$; flip angle $90^{\circ}$; field of view $192 \mathrm{~mm} \times 192 \mathrm{~mm}^{2}$; matrix size $64 \times 64$; voxel size $3 \times 3 \times 3 \mathrm{~mm}$. Field maps were collected in order to remove distortion caused by magnetic field inhomogeneity. Stimuli were projected centrally onto a screen at the front of the magnet, which

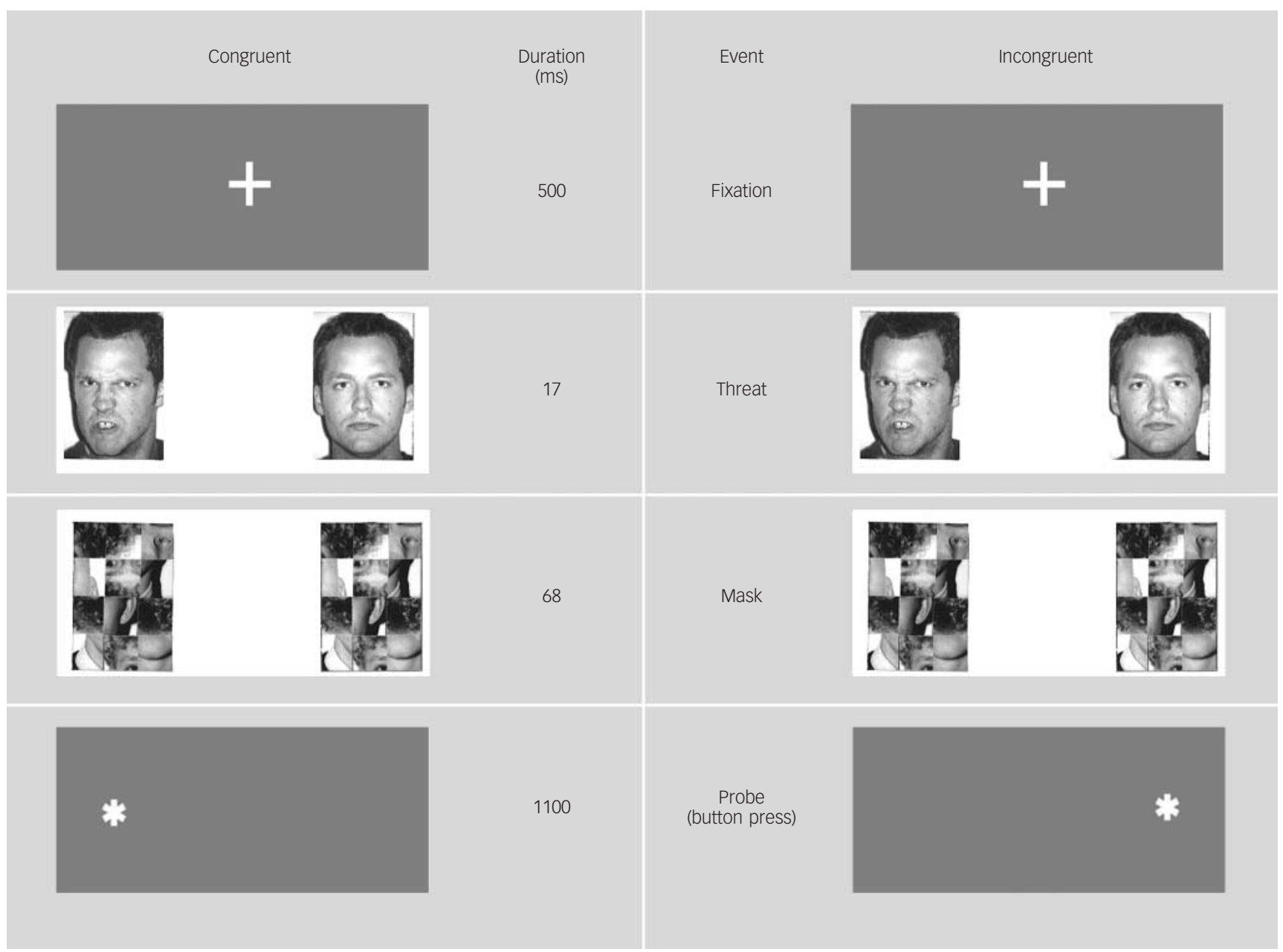

Fig. 1 Examples of incongruent and congruent masked angry-neutral trials presented with the duration and name of each event for the two types of trials.

Happy-neutral and neutral-neutral trials (not shown) were also presented to participants. 
participants viewed using a mirror mounted on the head coil $\left(21 \times 13^{\circ}\right.$ of visual angle of the whole screen $)$.

The images were pre-processed and subsequently analysed using SPM8 (www.fil.ion.ucl.ac.uk/spm/software/spm8), implemented in MATLAB 7.5 on Windows XP. After discarding the first six functional volumes of each session to allow for $T_{1}$ equilibrium, EPI images were spatially realigned to the first volume of the first run to correct for motion artefacts. These images were also corrected for geometric distortions caused by susceptibilityinduced field inhomogeneities. The field maps were processed for each participant's run using the FieldMap toolbox implemented in SPM8 to produce a voxel displacement map indicating the field distortions. The EPI images were then unwarped using the voxel displacement maps, normalised into standard anatomical space defined by the Montreal Neurological Institute (MNI) with a resampled voxel size of $3 \times 3 \times 3 \mathrm{~mm}$, and smoothed with an $6 \mathrm{~mm}$ full-width at half maximum Gaussian kernel filter. In line with Monk et al, ${ }^{16}$ trials with incorrect behavioural responses or responses that were less than $200 \mathrm{~ms}$ or greater than $1100 \mathrm{~ms}$ were removed from fMRI analysis. The maltreated group had a mean of $7.1 \%$ (s.d. $=5.4 \%$ ) of incorrect trials, whereas the comparison group had a mean of $4.4 \%$ (s.d. $=5.6 \%)$ of incorrect trials. Groups did not differ in the number of incorrect trials, $t(39)=-1.57$, $P=0.12$.

After pre-processing, the smoothed, normalised functional imaging data were entered into a voxel-wise participant-specific general linear model (GLM) with five regressors for the masked faces (angry-neutral congruent, angry-neutral incongruent, happy-neutral congruent, happy-neutral incongruent, neutralneutral) and one regressor for the blank trials. Trials within each of these six regressors were modelled as stick functions based on trial onset, convolved with a canonical haemodynamic response function. In addition, to correct for residual effects of head motion, six participant-specific movement parameters (derived from the realignment phase of pre-processing) were included as regressors of no interest. An additional regressor of no interest was included to model nuisance trials (i.e. trials that contained incorrect responses, null responses or responses that were too fast or too slow). For 22 participants (11 in the maltreated group and 11 in the non-maltreated group) an extra regressor was included to model a small number of corrupted images resulting from motion greater than $1.5 \mathrm{~mm}$ (half the size of our voxel size acquisition). These images ( $\leqslant 10 \%$ of each participant's data) were removed and the adjacent images interpolated in order to prevent distortion of the between-participant mask. To remove low-frequency drifts, data were high-pass filtered using a set of discrete cosine basis functions with a cut-off period of $128 \mathrm{~s}$.

The parameter estimates were calculated for all brain voxels using the GLM. Preliminary analyses revealed no effects of congruency, and so reported analyses collapse across congruent and incongruent trials for each emotion. Contrast images for 'angry $>$ neutral' (i.e. the combination of angry-neutral congruent and angry-neutral incongruent $>$ neutral-neutral) and 'happy $>$ neutral' (i.e. the combination of happy-neutral congruent and happy-neutral incongruent $>$ neutral-neutral) were computed in participant-specific fashion. Next, the participant-specific contrast images were entered into separate second-level analyses for each contrast of interest, where group (maltreated group, nonmaltreated group) served as a between-participants variable in independent sample $t$-tests. The interaction between group and emotion for each contrast were then explored. The amygdala, our a priori region of interest, was anatomically defined based on the automated anatomical labelling bilateral mask from the WFU PickAtlas. ${ }^{33}$ Within the amygdala mask we report results reaching significance at $P<0.05$, family-wise error (FWE) corrected. Exploratory correlational analyses were conducted in SPSS version 19 on Windows XP to examine potential associations between brain activation and (a) maltreatment indices (severity, duration, age at onset) and (b) psychiatric symptoms for those indices where significant group differences or strong trends (i.e. $P<0.1)$ were observed. Peak voxel data were used as they reflect a weighted average of the surrounding voxels due to smoothing. Parametric and non-parametric correlations were used for normally distributed and non-normally distributed data, respectively. Finally, we also examined trends for group differences at whole brain level using a statistical threshold of $P<0.001$ (uncorrected) with an extent threshold of $k \geqslant 4$ voxels, calculated according to the theory of Gaussian random fields.

\section{Analysis of behavioural and questionnaire data}

Criteria for determining the acceptability of trials in the behavioural analysis were the same as for the fMRI data analysis. Attention bias scores were derived from a standard formula, which involves subtracting for each participant the mean reaction time on trials where the emotion (angry or happy) face and probe appeared on the same side of the screen (congruent trials) from the mean reaction time on trials where the emotion face and probe appeared on the opposite side of the screen (incongruent trials). Positive bias scores (longer reaction times in congruent than incongruent trials) reflect the tendency to monitor the emotional stimulus, whereas negative bias scores reflect the tendency to avoid the emotional stimulus.

\section{Results}

\section{Psychiatric symptom scores}

The maltreated and non-maltreated groups did not differ on levels of STAIC anxiety and MFQ depression symptoms (Table 3). This was also reflected in the comparable levels of emotional symptoms across groups, reported by parents and carers on the SDQ. The groups differed, however, in relation to conduct problems and hyperactivity symptoms, with the maltreated sample showing on average higher levels of symptoms than their peers. Group differences in relation to levels of TSCC dissociation symptoms and PTSD symptoms were observed at trend level only (Table 3 ).

\section{Attentional bias on experimental paradigm}

There was no statistically significant group difference in terms of attentional bias and reaction time to either angry or happy trials (Table 4). A repeated measures ANOVA indicated no significant main effects or interactions (group $F(1,39)=0.25, \quad P=0.62$; emotion $F(1,39)=0.22, \quad P=0.65 ; \quad$ congruency $F(1,39)=1.43$, $P=0.24$; emotion $\times$ congruency $F(1,40)=0.10, P=0.75)$. Within each group separately there were no significant effects of congruency.

\section{Brain activity}

As hypothesised, the maltreated group, compared with the nonmaltreated group, exhibited greater activation in the right amygdala $(x=+18, \quad y=-1, \quad z=-17 ; Z=3.33 ; k=2 ; P=0.026$ FWE corrected) when masked angry and neutral faces were contrasted (Fig. 2a). However, compared with the non-maltreated group, the maltreated group also exhibited greater activation in the right amygdala $(x=+21, y=-1, z=-17 ; Z=3.20 ; k=3$; $P=0.039$ FWE corrected) when masked happy and neutral faces were contrasted (Fig. 2b). Trends for group differences in activation outside of our amygdala region of interest are shown in Table 5. 


\begin{tabular}{|c|c|c|c|}
\hline & \multicolumn{2}{|c|}{ Mean (s.d.) } & \multirow[b]{2}{*}{$P$} \\
\hline & Non-maltreated group $(n=23)$ & Maltreated group $(n=18)$ & \\
\hline \multicolumn{4}{|l|}{ Child rated } \\
\hline Mood and Feelings Questionnaire, total score & $11.70(7.90)$ & $11.17(9.17)$ & 0.85 \\
\hline \multicolumn{4}{|l|}{ Trauma Symptom Checklist for Children } \\
\hline Anxiety & $46.95(12.03)$ & $47.06(13.69)$ & 0.98 \\
\hline Depression & $44.68(9.38)$ & $45.53(12.23)$ & 0.81 \\
\hline Anger & $43.32(7.83)$ & $46.65(10.77)$ & 0.27 \\
\hline Post-traumatic stress & $44.50(6.30)$ & $49.53(11.60)$ & 0.09 \\
\hline Dissociation & $46.32(6.40)$ & $51.76(10.99)$ & 0.06 \\
\hline \multicolumn{4}{|l|}{ State-Trait Anxiety Inventory for Children } \\
\hline Trait & $32.61(7.68)$ & $32.24(8.58)$ & 0.89 \\
\hline State & $27.57(4.53)$ & $25.76(2.82)$ & 0.16 \\
\hline Total & $60.17(10.29)$ & $58.69(9.78)$ & 0.65 \\
\hline \multicolumn{4}{|l|}{ Parent rated } \\
\hline \multicolumn{4}{|l|}{ Strengths and Difficulties Questionnaire } \\
\hline Emotional symptoms score & $2.83(1.78)$ & 2.78 (1.59) & 0.93 \\
\hline Conduct problems score & $1.48(1.24)$ & $3.44(2.28)$ & 0.00 \\
\hline Hyperactivity score & $3.43(2.76)$ & $5.57(3.04)$ & 0.02 \\
\hline Peer problems score & $2.00(1.73)$ & $1.61(1.98)$ & 0.51 \\
\hline Prosocial behaviour score & $8.26(2.38)$ & $7.99(1.96)$ & 0.70 \\
\hline
\end{tabular}

\begin{tabular}{|c|c|c|c|}
\hline \multirow[b]{2}{*}{ Masked emotional faces } & \multicolumn{2}{|c|}{ Mean (s.d.) } & \multirow[b]{2}{*}{$P$} \\
\hline & Non-maltreated group $(n=23)$ & Maltreated group $(n=18)$ & \\
\hline Angry faces attentional bias ${ }^{b}$ & $-1.1(52.5)$ & $-12.8(39.6)$ & 0.44 \\
\hline \multicolumn{4}{|c|}{ Angry-neutral comparison reaction times } \\
\hline Congruent & $609.1(69.4)$ & $618.1(49.1)$ & 0.73 \\
\hline Incongruent & $608.7(60.1)$ & $612.3(50.9)$ & 0.73 \\
\hline Happy faces attentional bias ${ }^{c}$ & $-10.6(38.4)$ & $1.7(54.8)$ & 0.40 \\
\hline \multicolumn{4}{|c|}{ Happy-neutral comparison reaction times } \\
\hline Congruent & $611.6(63.4)$ & $616.8(58.5)$ & 0.67 \\
\hline Incongruent & $606.3(66.1)$ & $617.5(49.9)$ & 0.67 \\
\hline $\begin{array}{l}\text { a. All values are in milliseconds. } \\
\text { b. When groups were analysed tog } \\
\text { c. When groups were analysed tog }\end{array}$ & $\begin{array}{l}\text { condition, } t(40)=-0.85, P=0.40 \\
\text { condition, } t(40)=-0.73, P=0.47\end{array}$ & & \\
\hline
\end{tabular}

\section{Correlational analyses}

No significant correlations were found between amygdala activation and attentional bias scores in either group. However, in the maltreated group several significant associations were observed between amygdala activation and indices of maltreatment experience (Fig. 3). Amygdala response to angry faces was negatively associated with age at onset of emotional maltreatment $\left(r_{\mathrm{s}}=-0.70, P=0.005\right.$; Fig. 3a) and age at onset of neglect $\left(r_{\mathrm{s}}=-0.72, P=0.004\right.$; Fig. $\left.3 \mathrm{~b}\right)$, implying that heightened amygdala response was associated with an earlier onset of these maltreatment subtypes. Consistent with this finding, duration of emotional maltreatment was positively associated with amygdala activation to angry faces $\left(r_{\mathrm{s}}=0.75, P=0.001\right.$; Fig. $\left.3 \mathrm{c}\right)$ and happy faces $\left(r_{\mathrm{s}}=0.72, P=0.003\right.$; Fig. $\left.3 \mathrm{~d}\right)$. No significant associations were observed between amygdala activation (for either angry or happy faces) and psychiatric symptoms. However, a few trend-level associations were found (amygdala activation to angry faces and PTSD $(r=0.47, P=0.06)$ and dissociation symptoms $(r=0.46, P=0.06)$; amygdala activation to happy faces and dissociation symptoms $(r=0.46, P=0.06))$. All significant correlations reported above and depicted in Fig. 3 remained significant when PTSD and dissociation symptoms were included as covariates of no interest in partial correlation analyses.

\section{Discussion}

Our aim was to investigate the impact of maltreatment on preattentive processing of emotional cues in a community sample of children. Specifically, we used an established paradigm to investigate neural response to angry and happy faces. ${ }^{16}$ As predicted, we found that maltreated children, compared with matched peers, showed greater activation in the right amygdala when processing angry faces. However, contrary to our original predictions, we also found elevated right amygdala activation in the maltreated group for happy faces. These findings suggest that maltreatment in the home is associated with alterations even in the earliest stages of affect processing to both positive and negative facial affect.

The increased activation of the amygdala in response to angry faces in the maltreated children is in line with previous ERP and fMRI findings that point to a pattern of 'hypervigilent' response to threat-related cues in children exposed to different forms of early adversity. ${ }^{4,13}$ The right-lateralised amygdala group finding is also in line with most previous effects reported for masked emotional stimuli. ${ }^{34}$ The current findings extend previous ERP and fMRI work in maltreated children and point to a pattern of atypical neural processing of threat cues even outside of conscious 
awareness. However, we also found heightened neural response to happy faces, which appears somewhat at odds with possible adaptation to 'threat-specific' cues only. ${ }^{15,16}$ One possibility is that previous studies have tended to use paradigms in which faces were presented for extended durations; by contrast, the current paradigm, by using pre-attentive presentation of faces, is likely to have indexed earlier stages of processing that are less amenable to higher-order strategic or regulatory influences. Future studies are required to explore whether differential response to pre-attentively presented happy faces has experiential or clinical sequalae.

This pattern of heightened amygdala activation to both happy and angry faces in maltreated children points to broader alterations in affect processing than previously thought. Specifically, these findings suggest that maltreatment heightens neural response to emotional valence (either positive or negative) during the very early stages of facial processing, whereas a selective response to threat (such as anger) may only characterise later stages of processing. ${ }^{4}$ Future studies using magnetoencephalography (MEG) may be helpful in delineating the time course of such processes. Our finding of significant associations between maltreatment experience and neural activation are consistent with the view that heightened amygdala activation to facial affect may represent an adaptation to environmental stress. In relation to emotional abuse, for example, earlier age at onset and longer duration of abuse were associated with greater neural response in the amygdala to angry faces. This finding suggests that

(a)

$$
\text { Angry }>\text { neutral }
$$

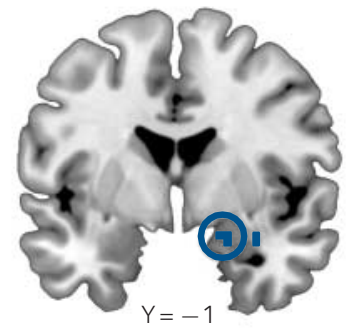

(b) Happy > neutral

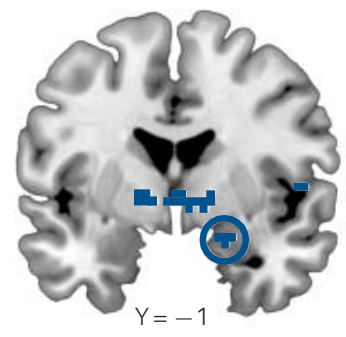

Fig. 2 Greater right amygdala activation to masked angry and masked happy faces relative to neutral faces in the maltreated group.

(a) Statistical parametric map (SPM) showing increased right amygdala activation in maltreated children for the contrast angry $>$ neutral $(x, y, z$ coordinates: $18,-1,-17$; maltreated children for the contrast angry $>$ neutral $(x, y, z$ coordinates: $18,-1$,
$Z=3.33 ; k=2 ; P=0.026$, family-wise error (FWE) corrected). (b) Increased right $Z=3.33 ; k=2 ; P=0.026$, family-wise error (FWE) corrected). (b) Increased right
amygdala activation in maltreated children for the contrast happy $>$ neutral $(x, y, Z$ amygdala activation in maltreated children for the contrast happy $>$ neutral $(x, y$,
coordinates: $21,-1,-17 ; Z=3.20 ; P=0.039 ; k=3$; FWE corrected. SPMs are coordinates: $21,-1,-17 ; Z=3.20 ; P=0.039 ; k=3$; FWE corrected. SPMs are
thresholded at $P<0.005$ (uncorrected) for visualisation purposes and all coordinates reference the coordinate system of the Montreal Neurological Institute. amygdala activation is to some degree calibrated in line with length of exposure to environmental stress, with exposure during the first 2 years of life appearing particularly influential, consistent with related findings regarding amygdala structure. ${ }^{35}$

In considering the research literature more broadly, it has been proposed that early detection of salient emotional cues accords functional and survival advantages ${ }^{36}$ and there is now substantial evidence from human neuroimaging studies for a largely subcortical system that responds to pre-consciously processed emotional signals. ${ }^{15}$ Several studies investigating clinical populations have reported heightened responsiveness of this subcortical system during early stages of affect processing. For example, adult soldiers with PTSD show greater amygdala response to pre-attentively presented threat cues, ${ }^{37,38}$ as do children and adolescents with generalised anxiety disorder. ${ }^{16}$ We speculate that such heightened activation may represent an adaptation to environmental adversity conferring short-term functional advantages, for example improving the ability to rapidly detect affective cues in an abusive home environment. However, any such adaptation may incur longer-term costs for the child, limiting attentional resources for mastering age-appropriate skills in social and academic domains. In addition, heightened neural response to affect may increase vulnerability to psychopathology in the longer term. ${ }^{4,39}$

Although we have focused on atypical amygdala activation in line with previous studies of childhood adversity, ${ }^{4,10,11}$ we also present preliminary evidence for atypical activation in a number of cortical and subcortical regions implicated in pre-attentive processing, including the cerebellum, thalamus and pallidum.

\section{Limitations}

A limitation of the current study is the use of a cross-sectional design, which constrains our ability to draw causal inferences between maltreatment exposure and the observed patterns of atypical neural response. Longitudinal studies are required to address issues of causality and whether observed differences remain over time. Another limitation, inherent in studying typically heterogeneous community populations of maltreated children, ${ }^{40}$ is the difficulty in making specific inferences about individual forms of maltreatment. A third limitation relates to potential recruitment bias. Although the final sample included a number of families characterised by severe maltreatment histories, the voluntary nature of the recruitment process may have led to an underrepresentation of more disturbed and chaotic families. Finally, because of our sample size, we were unable to examine the influence of gender, which we know is associated with differential outcomes for boys and girls exposed to early adversity in general $^{41}$ and maltreatment in particular. ${ }^{24}$

\begin{tabular}{|c|c|c|c|c|c|c|}
\hline & $P$ uncorrected & $k \geqslant 4$ & Left/right & $\mathrm{BA}$ region & Z-value & Maximum z position (MNI) \\
\hline \\
\hline \multicolumn{7}{|l|}{ Angry $>$ neutral } \\
\hline Cerebellum & $<0.001$ & 4 & Left & - & 3.26 & $-15,-46,-23$ \\
\hline \multicolumn{7}{|l|}{ Happy $>$ neutral } \\
\hline Thalamus & $<0.001$ & 4 & Right & - & 3.72 & $9,-16,-2$ \\
\hline Pallidum & $<0.001$ & 11 & Right & - & 3.67 & $15,2,-2$ \\
\hline \multicolumn{7}{|l|}{ Maltreated $<$ non-maltreated } \\
\hline \multicolumn{7}{|l|}{ Happy $>$ neutral } \\
\hline Temporal pole/middle temporal gyrus & $<0.001$ & 12 & Left & 38 & 3.82 & $-39,8,-29$ \\
\hline Temporal pole/superior temporal gyrus & $<0.001$ & 5 & Right & 38 & 3.3 & $33,14,-26$ \\
\hline
\end{tabular}


(a)

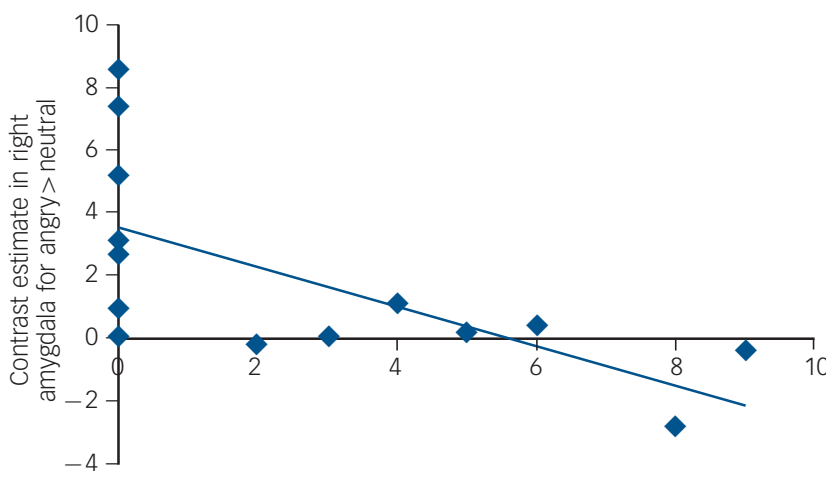

(C)

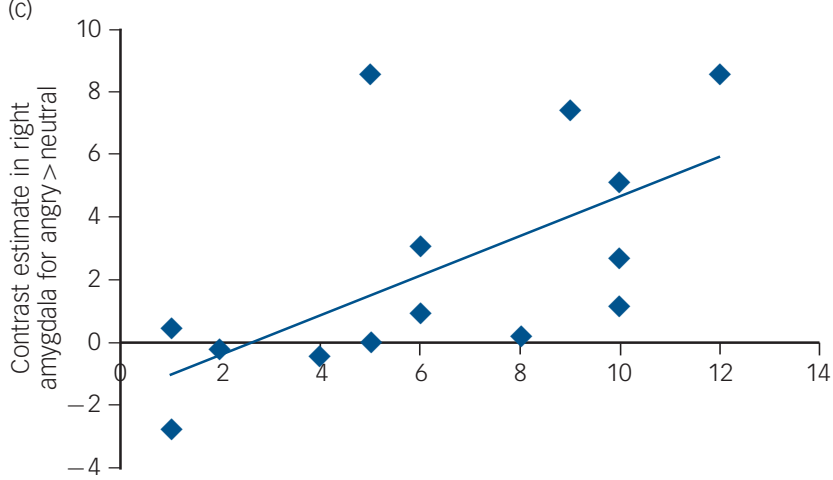

(b)

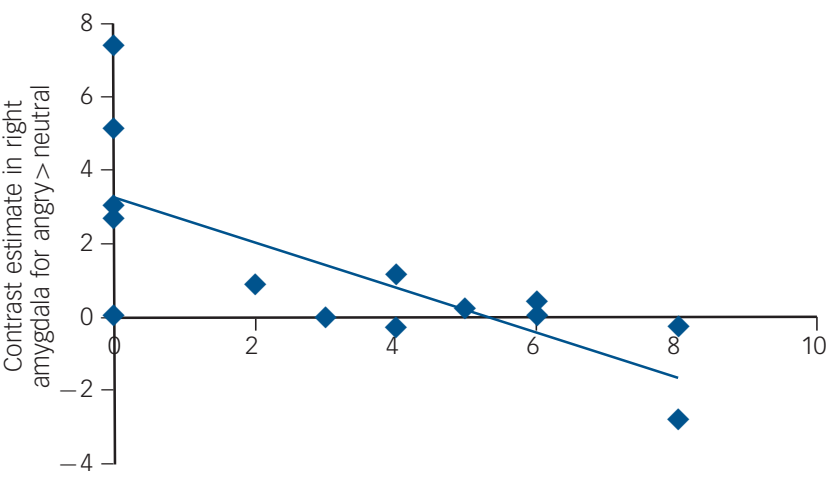

(d)

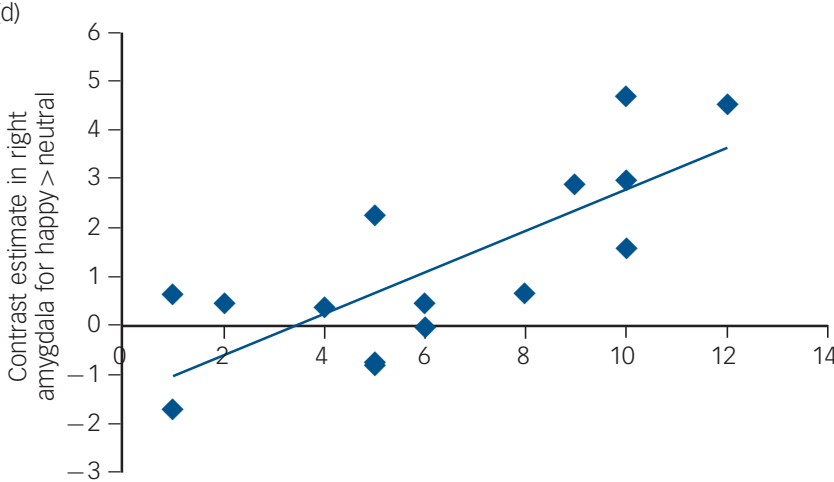

Fig. 3 Scatter plots depicting the correlations between the contrast estimates (angry $>$ neutral) from the right amygdala and (a) age at onset of emotional abuse, (b) age at onset of neglect, and (c) duration of emotional abuse. (d) Scatter plot depicting the correlations between the contrast estimates (happy > neutral) from the right amygdala and duration of emotional abuse.

Seventeen maltreated children were identified as having been exposed to emotional abuse, but information about age at onset was only available for 14 participants, whereas information about duration was only available for 15 participants (note that for the plots depicting duration in panels (c) and (d), two data points overlap for 5 years' duration).

\section{Implications}

In conclusion, these findings suggest that exposure to maltreatment in the home is associated with an atypical pattern of neural adaptation. ${ }^{4,13}$ We demonstrate for the first time that childhood maltreatment is associated with increased amygdala activation to pre-attentively presented positive and threatening faces. In other words, maltreated children show a general pattern of atypical affect processing, even outside of conscious awareness. Given the evidence that pre-attentive affect processing is also altered in individuals with anxiety disorders, we suggest that this pattern of heightened amygdala activation in maltreated children may constitute a latent neural risk marker, associated with increased vulnerability to future psychiatric disorder. ${ }^{4}$

Eamon J. Mccrory, PhD, DClinPsy, Division of Psychology and Language Sciences, University College London and The Anna Freud Centre, London; Stéphane A. De Brito, PhD, School of Psychology, University of Birmingham; Philip A. Kelly, MSc, Division of Psychology and Language Sciences, University College London and The Anna Freud Centre, London; Geoffrey Bird, PhD, Department of Psychological Sciences, Birkbeck College, London, and Institute of Cognitive Neuroscience, University College London; Catherine L. Sebastian, PhD, Division of Psychology and Language Sciences, University College London; Andrea Mechelli, PhD, Department of Psychosis Studies, Institute of Psychiatry, King's College London; Sophie Samuel, MSC, Essi Viding, PhD, Division of Psychology and Language Sciences, University College London, UK

Correspondence: Eamon J. McCrory, Division of Psychology and Language Sciences, University College London, 26 Bedford Way, London WC1H OAP, UK. Email: e.mccrory@ucl.ac.uk

First received 20 Jun 2012, final revision 17 Jan 2013, accepted 17 Jan 2013

\section{Funding}

This work was supported by a grant from the U.K. Economic and Social Research council (RES-061-25-0189) to E.J.M.

\section{Acknowledgements}

We thank the children, parents, carers and social workers who generously participated in this research, and Helen Maris, who helped with the data collection. We also thank Karin Mogg and Brendan Bradley for kindly sharing their stimuli with us.

\section{References}

1 Gilbert R, Widom CS, Browne K, Fergusson D, Webb E, Janson S. Burden and consequences of child maltreatment in high-income countries. Lancet 2009; 373: 68-81.

2 Scott KM, Smith DR, Ellis PM. Prospectively ascertained child maltreatment and its association with DSM-IV mental disorders in young adults. Arch Gen Psychiatry 2010; 67: 712-9.

3 Carrion VG, Haas BW, Garrett A, Song S, Reiss AL. Reduced hippocampal activity in youth with posttraumatic stress symptoms: an fMRI study. J Pediatr Psychol 2010; 35: 559-69.

4 Mccrory EJ, De Brito SA, Sebastian CL, Mechelli A, Bird G, Kelly PA, et al. Heightened neural reactivity to threat in child victims of family violence. Curr Biol 2011; 21: R947-8.

5 Pollak SD, Klorman R, Thatcher JE, Cicchetti D. P3b reflects maltreated children's reactions to facial displays of emotion. Psychophysiology 2001; 38 : 267-74. 
6 McCrory E, De Brito SA, Viding E. The impact of childhood maltreatment: a review of neurobiological and genetic factors. Front Psychiatry 2011; 2: 48

7 Aue T, Lavelle LA, Cacioppo JT. Great expectations: what can fMRI research tell us about psychological phenomena? Int J Psychophysiol 2009; 73: 10-6.

8 Etkin A, Wager TD. Functional neuroimaging of anxiety: a meta-analysis of emotional processing in PTSD, social anxiety disorder, and specific phobia. Am J Psychiatry 2007; 164: 1476-88.

9 Van Wingen GA, Geuze E, Vermetten E, Fernández G. Perceived threat predicts the neural sequelae of combat stress. Mol Psychiatry 2011; 16 664-71.

10 Maheu FS, Dozier M, Guyer AE, Mandell D, Peloso E, Poeth K, et al. A preliminary study of medial temporal lobe function in youths with a history of caregiver deprivation and emotional neglect. Cogn Affect Behav Neurosci 2010; 10: 34-49.

11 Tottenham N, Hare TA, Millner A, Gilhooly T, Zevin JD, Casey BJ. Elevated amygdala response to faces following early deprivation. Dev Sci 2011; 14: 190-204.

12 Rutter M, Sonuga-Barke EJ, Castle J. I: Investigating the impact of early institutional deprivation on development: background and research strategy of the English and Romanian adoptees (ERA) study. Monogr Soc Res Child 2010; 75: 1-20.

13 Pollak SD. Mechanisms linking early experience and the emergence of emotions: illustrations from the study of maltreated children. Curr Dir Psychol Sci 2008; 17: 370-5.

14 Cicchetti D, Curtis WJ. An event-related potential study of the processing of affective facial expressions in young children who experienced maltreatment during the first year of life. Dev Psychopathol 2005; 17: 641-77.

15 Tamietto M, De Gelder B. Neural bases of the non-conscious perception of emotional signals. Nat Rev Neurosci 2010; 11: 697-709.

16 Monk CS, Telzer EH, Mogg K, Bradley BP, Mai X, Louro HMC, et al. Amygdala and ventrolateral prefrontal cortex activation to masked angry faces in children and adolescents with generalized anxiety disorder. Arch Gen Psychiatry 2008; 65: 568-76.

17 Etkin A, Klemenhagen KC, Dudman JT, Rogan MT, Hen R, Kandel ER, et al. Individual differences in trait anxiety predict the response of the basolateral amygdala to unconsciously processed fearful faces. Neuron 2004; 44: 1043-55.

18 Armony JL, Corbo V, Clément $\mathrm{MH}$, Brunet A. Amygdala response in patients with acute PTSD to masked and unmasked emotional facial expressions. Am J Psychiat 2005; 162: 1961-3.

19 Bryant RA, Kemp AH, Felmingham KL, Liddell B, Olivieri G, Peduto A, et al. Enhanced amygdala and medial prefrontal activation during nonconscious processing of fear in posttraumatic stress disorder: an fMRI study. Hum Brain Mapp 2008; 29: 517-23.

20 Frewen PA, Dozois DJA, Joanisse MF, Neufeld RW. Selective attention to threat versus reward: meta-analysis and neural-network modeling of the dot-probe task. Clin Psychol Rev 2008; 28: 308-38.

21 Dodge KA, Bates JE, Pettit GS. Mechanisms in the cycle of violence. Science 1990; 250: 1678-83.

22 Magdol L, Moffitt TE, Caspi A, Silva PA. Developmental antecedents of partner abuse: a prospective-longitudinal study. J Abnorm Psychol 1998; 107 375-89
23 Kaufman J, Jones B, Stieglitz E, Vitulano L, Mannarino AP. The use of multiple informants to assess children's maltreatment experiences. J Fam Violence 1994; 9: 227-48.

24 Lansford JE, Dodge KA, Pettit GS, Bates JE, Crozier J, Kaplow J. A 12-year prospective study of the long-term effects of early child physical maltreatment on psychological, behavioral, and academic problems in adolescence. Arch Pediat Adol Med 2002; 156: 824-30.

25 Straus MA. Measuring intrafamily conflict and violence: the Conflict Tactics (CT) Scales. In Physical Violence in American Families: Risk Factors and Adaptations to Violence in 8,145 Families (eds MA Straus, RJ Gelles): 29-47. Transaction, 1990.

26 Wechsler D. Wechsler Abbreviated Scale of Intelligence. The Psychological Corporation, 1999

27 Spielberger CD. Manual for the State-Trait Anxiety Inventory for Children (Form Y). Consulting Psychologists Press, 1973.

28 Angold A, Costello EJ, Messer SC. Development of a short questionnaire for use in epidemiological studies of depression in children and adolescents. Int J Method Psych 1996; 5: 237-49.

29 Briere J. Trauma Symptom Checklist for Children (TSCC). Psychological Assessment Resources, 1996

30 Goodman R. The Strengths and Difficulties Questionnaire: a research note. J Child Psychol Psychiatry 1997; 38: 581-6.

31 Mogg K, Bradley BP. Selective orienting of attention to masked threat faces in social anxiety. Behav Res Ther 2002; 40: 1403-14.

32 Weiskopf N, Hutton C, Josephs O, Deichmann R. Optimal EPI parameters for reduction of susceptibility-induced BOLD sensitivity losses: a whole-brain analysis at $3 \mathrm{~T}$ and $1.5 \mathrm{~T}$. Neuroimage 2006; 33: 493-504.

33 Maldjian JA, Laurienti PJ, Kraft RA, Burdette JH. An automated method for neuroanatomic and cytoarchitectonic atlas-based interrogation of fMRI data sets. Neuroimage 2003; 19: 1233-9.

34 Costafreda SG, Brammer MJ, David AS, Fu CHY. Predictors of amygdala activation during the processing of emotional stimuli: a meta-analysis of 385 PET and fMRI studies. Brain Res Rev 2008; 58: 57-70.

35 Tottenham N, Hare TA, Quinn BT, McCarry TW, Nurse M, Gilhooly T, et al. Prolonged institutional rearing is associated with atypically large amygdala volume and difficulties in emotion regulation. Dev Sci 2010; 13: 46-61.

36 LeDoux JE. The Emotional Brain. Simon \& Schuster, 1996

37 Rauch SL, Whalen PJ, Shin LM, McInerney SC, MacKlin ML, Lasko NB, et al. Exaggerated amygdala response to masked facial stimuli in posttraumatic stress disorder: a functional MRI study. Biol Psychiatry 2000; 47: 769-76.

38 Hendler $T$, Rotshtein $P$, Yeshurun $Y$, Weizmann $T$, Kahn I, Ben-Bashat $D$, et al. Sensing the invisible: differential sensitivity of visual cortex and amygdala to traumatic context. Neuroimage 2003; 19: 587-600.

39 Shackman JE, Shackman AJ, Pollak SD. Physical abuse amplifies attention to threat and increases anxiety in children. Emotion 2007; 7: 838-52.

40 Dong M, Anda RF, Felitti VJ, Dube SR, Williamson DF, Thompson TJ, et al. The interrelatedness of multiple forms of childhood abuse, neglect, and household dysfunction. Child Abuse Negl 2004; 28: 771-84.

41 Bos K, Zeanah CH, Fox NA, Drury SS, McLaughlin KA, Nelson CA. Psychiatric outcomes in young children with a history of institutionalization. Harv Rev Psychiatry 2011; 19: 15-24. 